\title{
A Battery-Less, Energy Harvesting Device for Long Range Scavenging of Wireless Power from Terrestrial TV Broadcasts
}

\author{
${\text { Rushi } \text { Vyas }^{* 1}, \text { Hiroshi Nishimoto }}^{2}$, Dr. Manos Tentzeris ${ }^{1}$, Dr. Yoshihiro Kawahara, ${ }^{1,2}$, Dr. Tohru Asami ${ }^{2}$ \\ ${ }^{1}$ School of Electrical and Computer Engineering, Georgia Institute of Technology \\ ${ }^{2}$ Graduate School of Information and Technology, University of Tokyo \\ *Rushi.Vyas@gatech.edu
}

\begin{abstract}
Use of Japanese Integrated Services Digital Broadcasting Terrestrial (ISDB-T) standard has resulted in broadcast of perpetually on, wireless digital TV signals over the air at wider bandwidths in UHF bands for smart phones \& videoon-demand. This paper presents a unique energy harvesting prototype capable of scavenging wireless power from such broadcasts and well capable of powering on electronics operating at $3 \mathrm{~V}$ from wireless $\mathrm{TV}$ signals for limited duty cycle at distance of $6.5 \mathrm{~km}$ from source.
\end{abstract}

Index Terms - Antenna, Charge Pump, DVT, Energy Harvesting, Power Scavenging, TV, Wireless Power transfer.

\section{INTRODUCTION}

The advent of the Japanese ISDB-T standard for broadcasting digital TV over the air since 1990s has resulted in huge data payloads broadcasted at multiple frequency bands over the air. Rather than carry video signals over a single frequency, digital streams are sent at digitally modulated, closely spaced carrier frequencies referred to as orthogonal frequency-division multiplexing (COFDM or OFDM) modulation, which allows for transmission of a much more enhanced picture quality without suffering ghosting and multipath effects prevalent with Analog TV. Peak digital transmit powers being lower than Analog, its broadcasting towers can be found at regular geographic intervals in most urban areas in order to maximize coverage. Ambient wireless power in the UHF bands due to digital TV broadcasts can offer a perpetual power source for sustaining battery-less, limited duty cycle operation of a number of low power embedded and wireless transceivers for autonomous wireless sensing with "smart skins"; implantable and wearable sensors for bio-monitoring; and "Internet of Things" applications. In this paper we present a unique prototype capable of scavenging wireless power from Digital TV broadcasts over ranges of kilometers for powering on low power embedded processors and wireless transceivers.

\section{AMBIENT WIRELESS ANALOG AND DIGITAL TV SIGNALS}

In order to maximize coverage and range, TV and Radio broadcast towers are spaced at regular geographic intervals in most urban areas, each broadcasting out between 10-100 kilowatts of effective radiated power (ERP) wirelessly in
Japan and the US. The amount of this power that can be captured using a polarization matched linear antenna on the receiver within a range of around 6.5 kilometers can be estimated to be in the 10 to-100s of micro-Watts range using Friis equation as in (1) [1] .

$$
P_{R}=P_{T} \cdot G_{T} \cdot G_{R} \cdot\left(\frac{\lambda}{4 \cdot \pi \cdot R}\right)^{2}
$$

Wireless power estimates at such long distances from the source were verified by ambient wireless spectrum measurements carried out at the point of interest in downtown Tokyo (POI) using a NARDA SRM-3000 Radiation meter, which show significant wireless activity in the VHF and UHF bands between 480 and $580 \mathrm{MHz}$ due to radio and TV broadcasts. The wireless spectrum measurement carried out at the POI at a distance of $6.5 \mathrm{~km}$ from TV broadcasts atop Tokyo tower is shown in Fig 1. The measured wireless signal levels due to Analog and Digital TV broadcasts in UHF bands in terms of their incident electric field intensity $(\mathrm{V} / \mathrm{m})$ are listed in table 1.

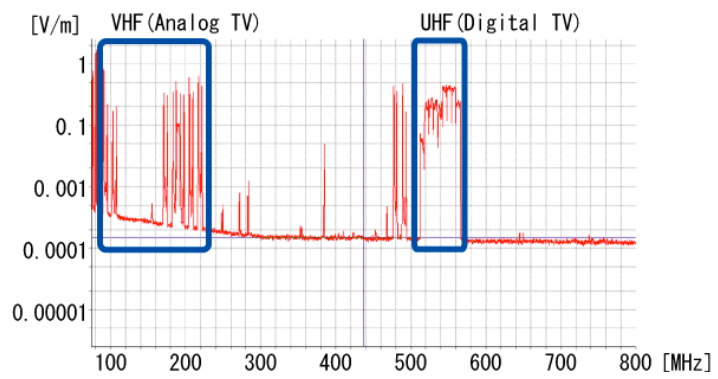

Fig. 1. Measured UHF and VHF TV and Radio Broadcasts measured in downtown Tokyo $-6.5 \mathrm{~km}$ from Tokyo TV Tower.

Harnessing enough energy out of such low amounts of incident wireless power in order to use it to power on electronics requires the design of an optimized antenna, an RF-to-DC charge pump circuit, a proper power matching between them to minimize reflection losses and a low leakage power management circuitry to keep the embedded end device from draining the charge tank super capacitor till it has reaches a high turn on voltage as in the system in Fig 2. 


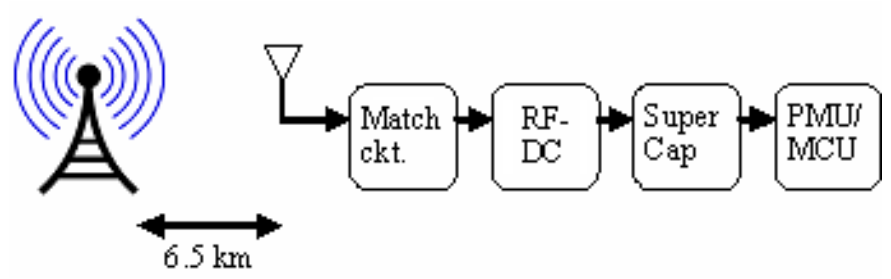

Fig. 2. System level description of energy harvesting device.

At long distances from the source, well into the far field range of the TV broadcasting antenna, the amount of useful power that can be harnessed from the incident Electric field in the UHF bands is a function of the antenna aperture area $\left(\mathrm{A}_{\mathrm{E}}\right)$, which is a function of the antenna gain $(\mathrm{G})$; its conjugate load matching with the RF to DC charge pump circuit ( ); the wavelength of the incident electric field; and any polarization mismatch losses either due to the antenna orientation. Since TV broadcasts are linear in polarization, linear antennas such as dipoles or monopoles or its arrays offer lower losses due to polarization mismatch and therefore present a larger Antenna aperture area to the incident wireless electric fields.

$$
P_{I N}=S \cdot A_{E}=\frac{\left|\vec{E}_{\theta}\right|^{2}}{\eta_{O}} \cdot G \cdot\left(1-|\Gamma|^{2}\right) \cdot\left(\frac{\lambda^{2}}{4 \cdot \pi}\right)
$$

For a half wavelength long antenna dipole, mounted horizontally in air well above the ground, the amount of RF power $\left(\mathrm{P}_{\mathrm{IN}}\right)$ that it can transduce from the incident Electric fields (E) measured at the POI can be determined using (2) as derived from [1] [2], and listed in Table 1. In (2) ' 。' is the free space electromagnetic wave impedance and ' $\mathrm{S}$ ' is the Poynting vector that gives the wireless power density in the air due the Electric fields. The maximum and minimum captured wireless power is due to measurement fluctuations observed in the Spectrum analyzer.

TABLE I

AMBIENT WIRELESS POWER MEASUREMENTS IN DOWNTOWN TOKYO DUE TO ANALOG \& DIGITAL TV BROADCASTS

\begin{tabular}{|l|c|c|c|}
\hline Freq. & Application & $\begin{array}{c}\text { Meas. Electric } \\
\text { Field Intensity } \\
\text { |E I }\end{array}$ & $\begin{array}{c}\text { Wireless Power } \\
\text { Capture by } \\
\text { Dipole } \mathbf{P}_{\text {IN }} \\
(\mathbf{M H z})\end{array}$ \\
\hline $560-580$ & Digital TV & $0.44-0.57$ & $18.54-30.76$ \\
$540-560$ & Digital TV & $0.62-0.79$ & $39.02-64.76$ \\
$520-540$ & Digital TV & $0.44-0.57$ & $21.44-35.58$ \\
$510-520$ & Digital TV & $0.007-0.01$ & $0.007-0.011$ \\
494 & Analog TV & $0.26-0.34$ & $8.88-14.74$ \\
487 & Analog TV & $0.62-0.80$ & $49.77-82.60$ \\
480 & Analog TV & $0.62-0.80$ & $51.23-85.03$ \\
\hline
\end{tabular}

\section{A. Dipole Antenna}

An optimized linear dipole antenna was designed and fabricated using an inkjet printing process to efficiently convert the incident Electric field hitting it at UHF bands into $\mathrm{RF}$ signal form to be fed into an impedance matched RF to DC charge pump circuit that stores the harnessed energy in the form of a charge across a low leakage super capacitor. The antenna length was optimized to be about half wavelength long to maximize its gain $(G)$ \& effective aperture area $\left(A_{e}\right)$, which would increase the voltage at its terminals and the incident wireless power collected as in (2). The antenna width was optimized to give it a wideband impedance of close to 50 ohms in order to minimize reflection losses with a matched RF to DC charge pump circuit. The measured $\left|S_{11}\right|$ of the antenna shows a reflection loss of lower than $-15 \mathrm{~dB}$ from $467 \mathrm{MHz}$ to well beyond $580 \mathrm{MHz}$, ensuring that $97 \%$ of the transduced wirelesses TV signal power makes it through to the matched RF to DC converter circuit as shown in Fig 3.

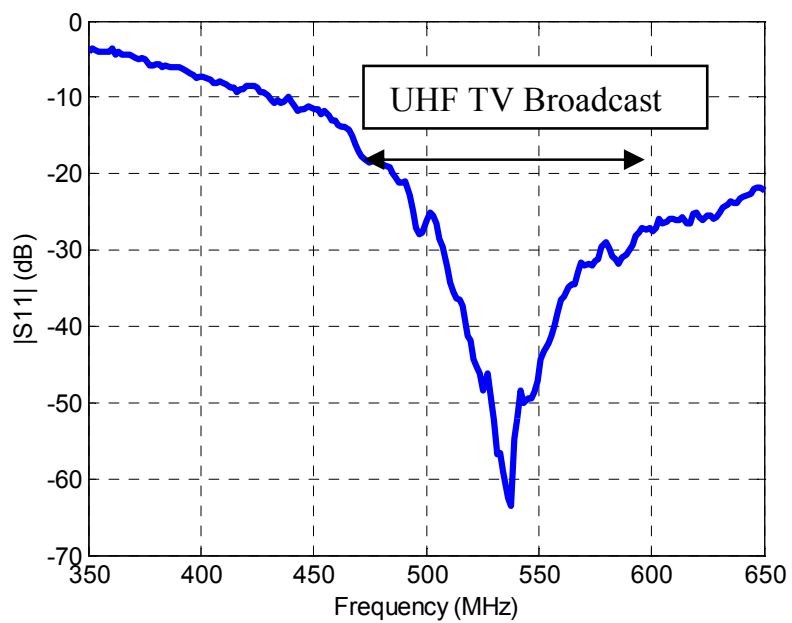

Fig. 3. Measured Dipole Antenna return loss.

\section{B. RF to DC Charge Pump Circuit}

The incoming Power transduced across the antenna is in RF form and induces a very low voltage across the antenna terminals. Being able to harness this power requires it to be rectified and stepped up to a voltage of $1.8 \mathrm{~V}$ or higher at which most electronic devices such as embedded processors and transceivers used for wireless sensing operate. A 5 stage RF charge pump circuit was designed and optimized for this task as shown in Fig 4 given its battery-less ability to step up and rectify the incoming RF voltage across the antenna compared to DC-DC power converters albeit with less gain control [3]. Low forward voltage Schottky diodes and proper circuit layout design help minimize circuit losses, turn on power and reverse leakage from the energy storing charge tank capacitor. Properly selected high Q capacitors used in the charge pump help minimize leakage and shorten the charge build up time at its output charge tank. A 100uF low leakage capacitor was used as the energy storage charge tank to power 
on the end device through hardware/firmware managed charge/discharge cycles similar to RFIDs.

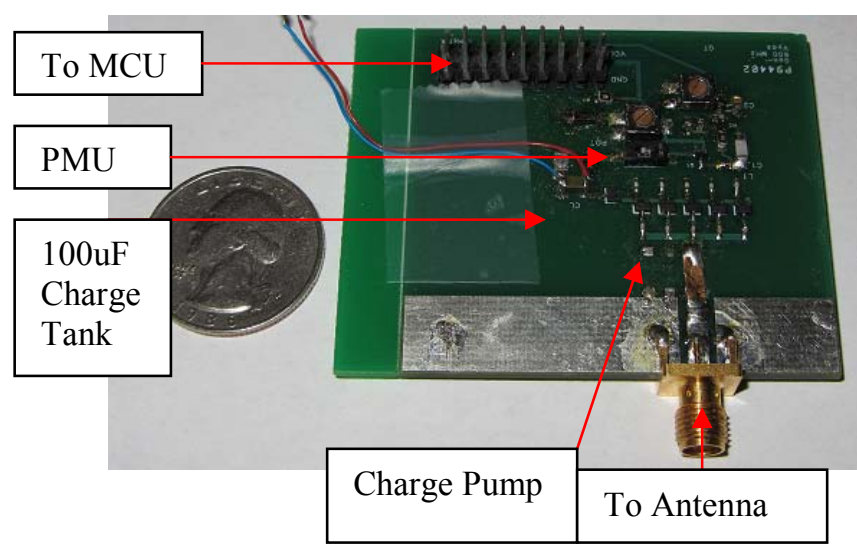

Fig. 4. Five stage Wireless Energy harvesting RF Charge Pump Circuit \& PMU.

The designed RF charge pump circuit exhibited very low power dissipative losses with an input impedance of about $0.52-\mathrm{j} 7.3 \mathrm{ohms}$ at UHF TV bands with the tank capacitor charged between 1.8 and 3.0V (VCAP). An optimal matching network was designed to match the $50 \mathrm{ohm}$ antenna impedance to the varying capacitive load of the Charge Pump Circuit across the digital UHF TV bands. The impedance matching maximizes the voltage at the charge pump input out of an already low input voltage at the antenna terminals which allows for wireless power to trickle into the charge tank at much lower input wireless power thereby maximizing the energy harvesting range of the system.

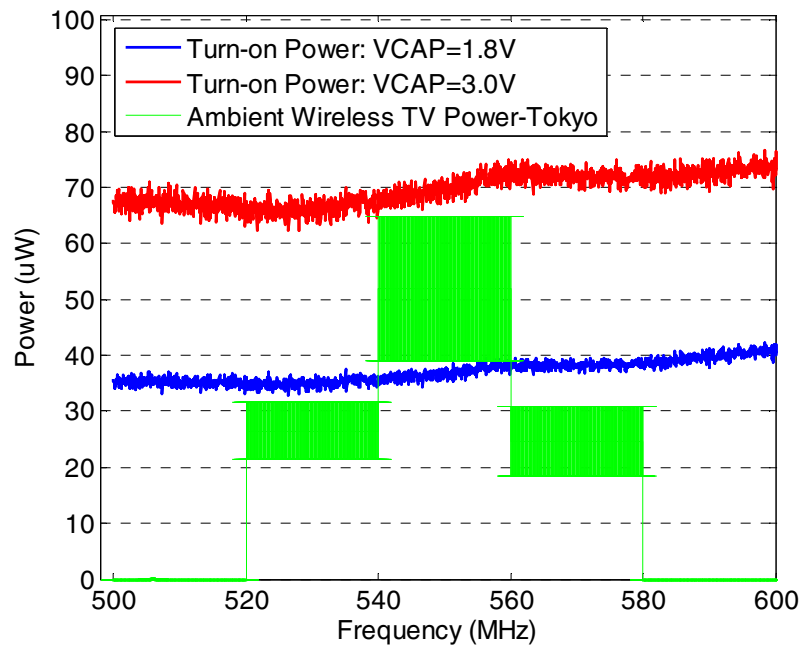

Fig. 5. Dipole captured wireless TV power \& required RF Charge Pump turn-on power to charge output tank capacitor to $1.8 \& 3.0 \mathrm{~V}$.

The amount of wireless power required by the Charge pump circuit to charge the tank capacitor to 1.8 and $3.0 \mathrm{~V}$ are 38 and 70 micro-watts respectively at $550 \mathrm{MHz}$, which is close to the wireless power captured by the antenna from the UHF TV bands at the POI as shown in Fig 5. Based on Fig 5, the energy harvesting circuit was expected to charge the charge tank close to $3 \mathrm{~V}$ at the POI which was verified by on field measurements as shown in Fig 6. At a distance of $6.5 \mathrm{~km}$ from Tokyo TV tower, the energy harvesting circuit charges up the $100 \mathrm{uF}$ charge tank to $2.9 \mathrm{~V}$ in 3 minutes making such a device ideal for battery less operation of wireless sensors for remote monitoring/sensing in most urban areas using just the existing terrestrial TV broadcast infrastructure for power.

\section{CONCLUSION}

An energy harvesting device was successfully designed to harvest power from ambient wireless TV signals at distance of over $6.5 \mathrm{~km}$ as demonstrated by field tests.
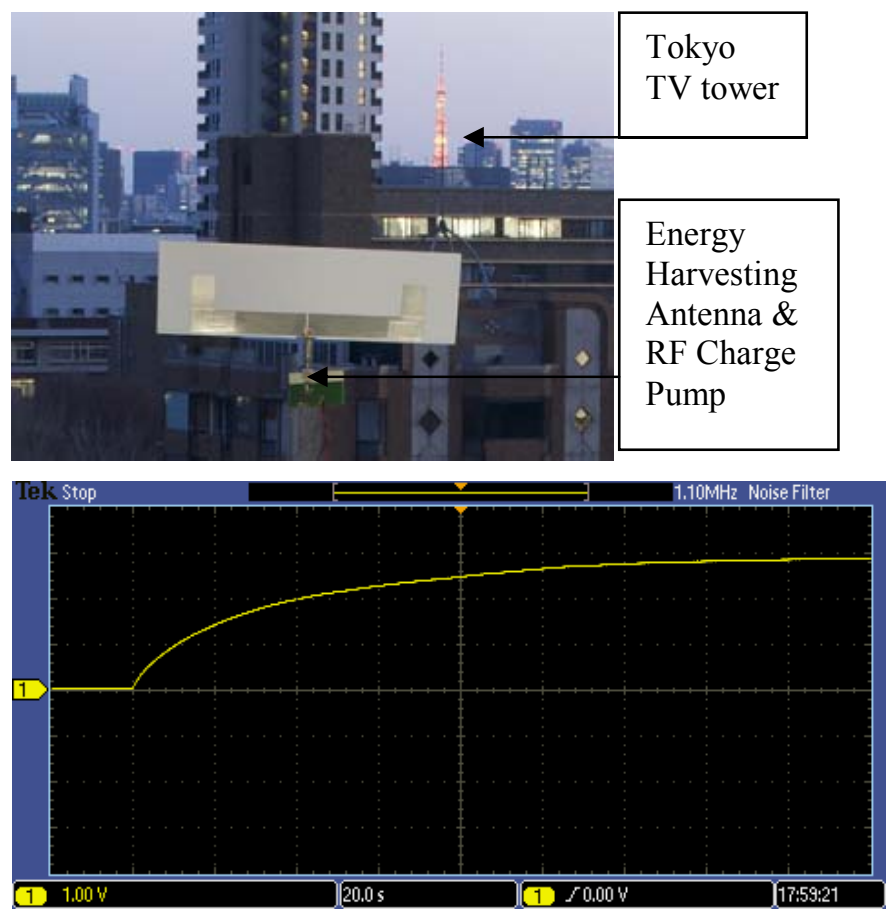

Fig. 6. Field Measurements of Energy harvesting device showing its charge tank capacitor charge to $2.9 \mathrm{~V}$ from wireless power harnessed from Tokyo TV Tower $6.5 \mathrm{~km}$ away.

\section{ACKNOWLEDGEMENT}

The authors wish to acknowledge NEDO Japan, NSF and the IFC alliance for their support.

\section{REFERENCES}

[1] C. Balanis, Antenna Theory. New York: Wiley, 1997. pp. 86.

[2] D.Pozar, Microwave Engineering. New York: Wiley, 2005. pp 21-25, 633-641.

[3] Dolgov et. al, "Power Management System for Online Low Power RF Energy Harvesting Optimization." IEEE

Transactions of Circuits \& Systems, vol. 57, issue 7, 2010. 